\title{
Persuasive Definitions: Values, Meanings and Implicit Disagreements
}

\author{
FABRIZIO MACAGNO
}

Università Cattolica del Sacro Cuore

Department of Linguistics

Via Necchi 5

20100 Milano

Italy

fabriziomacagno@hotmail.com

\section{DOUGLAS WALTON}

Centre for Research on Reasoning, Argumentation \& Rhetoric

University of Windsor

Windsor, $O N$

Canada N9B 3P4

dwalton@uwindsor.ca

\begin{abstract}
The purpose of this paper is to inquire into the relationship between persuasive definition and common knowledge (propositions generally accepted and not subject to dispute in a discussion). We interpret the gap between common knowledge and persuasive definition (PD) in terms of potential disagreements: PDs are conceived as implicit arguments to win a potential conflict. Persuasive definitions are analyzed as arguments instantiating two argumentation schemes, argument from classification and argument from values, and presupposing a potential disagreement. The argumentative structure of PDs reveals different levels of disagreement, and different possibilities of resolving the conflict or causing dialogical deadlock.
\end{abstract}

Resumé: Le but de cet article est d'examiner la relation entre une définition persuasive et des connaissances communes (les propositions généralement acceptées et qui ne sont pas sujettes à la contestation). Nous interprétons ce qui sépare les connaissances communes et les définitions persuasives (DP) en termes de désaccords potentiels : nous concevons des DP comme des arguments tacites qui visent à résoudre des conflits potentiels. Nous analysons les DP en termes d'arguments qui manifestent deux schèmes d'argumentation, des arguments fondés sur des classifications ou des valeurs, et qui présupposent un désaccord potentiel. La structure argumentative des DP revelent différents niveaux de désaccord et différentes possibilités de les résoudre ou de causer un dialogue inextricable

Keywords: clarification dialogues, communication failure, conflicts of meanings, conflicts of values, emotive meaning, quasi-definitions; value-based argumentation.

Stevenson (1938; 1944) introduced the notions of persuasive definition and quasi-definition. By the first term he meant the argumentative strategy of changing the denotative meaning of an emotive word in order to make it possible to predicate it of an object which otherwise would not be included in the extension of the term. By the second term he meant the 
technique of changing the emotional meaning of a term, leaving unaltered its denotative meaning. For instance, I can persuasively redefine 'culture' as originality, classifying in this fashion an original but illiterate person as cultured. In contrast, I can quasi-define 'blackguard' as meaning "the most interesting person, who does not live within the narrow limits of virtue” (see Stevenson, 1944, 280, 281). The first strategy is called persuasive definition, the second quasi-definition. We will refer to both as persuasive definitions (PDs), distinguishing the typologies by referring to Stevenson's distinction of types of meanings. Stevenson's proposal is highly interesting for argumentation, and opens up a series of problematic questions. Why is a term emotive? What are the strategies of redefinition? Up to what point is it possible and acceptable to redefine a term? What are the conditions of fallaciousness of persuasive definitions? Halldén (1960), Burgess-Jackson (1995), Aberdein (2000), and Schiappa (2003), to mention the most notable theories, described the types of redefinition manoeuvres, analysed the notion of definition showing its dependence on the theoretical background regarding the concept denoted by the definiendum, and inquired into the relation between vagueness and redefinition. In recent papers (Walton and Macagno 2007a; 2007b; 2007c) we analyzed what a definition is, what an emotive term is, and how an emotive term works in relation to redefinitions.

In this paper we want to highlight another aspect of persuasive and quasi-definitions. We want to examine why these types of redefinition are persuasive, by going into the relation between the interlocutors' common ground and the redefinition of the concept. In order for a definition to be a redefinition, in our perspective, it should not already be accepted by the interlocutors; or, better, it should not be an element of the common ground. The relation between definition, redefinition, and common ground is the object of this study. We want to inquire, in particular, into types of conflicts that can derive from a redefinition and the endoxic propositions it is grounded upon. Our purpose can be more simply described with an attempt to answer two questions. What and where is the difference of opinion or commitment in a redefinition? What is the possible object of conflict in a redefinition?

In the following sections we will explain the relation between redefinition and persuasion in terms of argumentation schemes, that is, common patterns of reasoning. A definition will be shown to be persuasive when it leads the interlocutor to accept a proposition or action, analyzed in terms of commitment, both action and propositional commitment (Walton and Krabbe 1995). The reasoning process a definition follows to achieve this result will be described by means of the arguments from classification and from values. Persuasive and quasidefinitions involve an appraisal of the aspect of reality they refer to, and a modification of the classificatory criteria. This classification and appraisal is the ground for a decision to act. We represent the connection between an evaluation of a fragment of reality and the decision to act accordingly as argument from values. 
Often persuasive definitions involve a conflict of values, in which the interlocutor founds his implicit argumentation upon a value that the interlocutor does not share. However, sometimes this conflict of values depends on the interlocutors' arguing about two different realities, two different concepts named in the same fashion. In order to understand and solve possible deadlocks, it is necessary to understand what kind of conflict is involved, whether of values, or of realities.

\section{Persuasive definitions: Types of "meaning" and types of conflicts}

In order to understand the types of disputes that can arise from PDs, it is useful to analyze how the two aspects of persuasive definitions, meaning and appraisal, can be objects of disagreement. In the following subsections the two meanings of PDs will be introduced, and the different ways in which they can controversial will be shown.

\subsection{Referential meaning and types of disagreement}

Persuasive definitions are essentially redefinitions. The theories mentioned in the introduction all deal with the problem of meaning, and try to explain why meaning can be crucial for persuasion. All the proposals advanced, in our view, can be seen as focussing on different aspects of meaning, more than exposing contradictory or incompatible approaches. Three fundamental concepts emerge from the accounts on persuasive definition: the crucial role of polisemy and ambiguity, the relationship between meaning and common ground, and the dependence of communication on shared knowledge.

The first aspect that needs to be analyzed in PDs is the relationship between a redefinition and the original meaning of a term. In Stevenson's perspective, PDs involve a change of the referential meaning of a term, leaving its emotional value unaltered. Stevenson's approach is basically behavioristic. We can interpret his explanation of PD as a technique aimed at introducing a new meaning while trading on the persisting effects of the old one. PDs, in our interpretation of Stevenson, are ways of introducing polisemy. Stevenson, however, does not distinguish between manipulative and allowed uses of redefinition. Burgess-Jackson and Aberdein focused on the conditions of admissibility of the redefinition. A word can be redefined when it is vague, that is, when its extensional boundaries are blurred. Some words have a kind of "grey zone" where it is not clear whether an object falls within the meaning of the word or not. A redefinition is in these cases useful to decide whether the objects belonging to a "grey zone" should be included in the extension of a concept or excluded from it.

An interesting aspect Burgess-Jackson highlights, and then Schiappa develops, is the dependence of definition on a theory. For instance, every definition of "rape" involves a particular sociological theory about the role of women in society and marriage (BurgessJackson1995, p. 426). Also, a definition of “death” involves a theory of 
life (see Schiappa, 2004). This factor raises a problem of primary importance for the study of meaning: the dependence of the definition of a word on more elementary concepts. In the Topics, one of the most crucial aspects of the Aristotelian definition is the notion of "things that are better known” (Topics VI, 4, 142a 5-15). A definition, in order to be a good definition, must start from what is better known, either relative to the interlocutor, or absolutely. We can interpret Burgess-Jackson and Schiappa's approach from this perspective. Definitions always involve more elementary concepts. For instance, death can be defined negatively by treating it as containing the components 'cessation' and 'life'. The conceptual elements used in a definition must be known by the interlocutors, that is, must be part of endoxa, or commonly shared body of propositions. A theory, in Schiappa's view is the underlying more elementary concepts a definition is made up of, combined in the definition. If the terms constituting the definition are ambiguous, the definition will be ambiguous too (see Topics, VI, 2, 139b 18-23). The polisemy of such terms used is not always evident. For instance, in his Nicomachean Ethics (1129a 25-30) Aristotle acknowledges that, in order to define 'injustice', it is necessary to show the subtly different meanings of 'justice'. Similarly, in order to define 'death', it is necessary to specify which meaning of 'life' is assumed, and, consequently, which meaning of 'death' is the concept defined.

The relation between common knowledge and polisemy is crucial to understand the origin of possible disagreements and disputes about the definition or use of terms. An extremely controversial (Clarke, 1979) work which offers insights on conflicts of opinion about meaning is Gallie's study on essentially contested concepts (1956). Gallie took into consideration some concepts, such as 'art' and 'champion'. He noticed that often when people use these terms, a disagreement can arise concerning their meaning. What counts as a "work of art", depends on artistic traditions, aesthetic values of a given culture, and differing styles that need to be taken into consideration. Similarly, what a "champion" is depends on what is considered a "good player". These concepts are essentially contested, since there is no agreement on what they mean. This observation opens up a series of problems. In first place, some controversial concepts seem to be polisemic terms. If a person uses the term 'art' to describe pornography (see also Schiappa for this example), it is likely that he is using a meaning of the term differently from the commonly shared use of the term. However, polisemy can also stem from an ambiguity in the defieniens' terms. If 'champion' is considered to be the best player, it is the notion of goodness and excellence that must be disambiguated. Moreover, ambiguity can depend on the fact that some terms are relative. In such cases, it is necessary to specify the argument constituting a predicate's semantic structure (see Rigotti, 2005). For instance, to define 'excellence' it might be necessary to specify the discipline in which a work or person is excellent (see also Aristotle, Topics VI, 6, 145a 12-25). Finally, terms can be vague, and the indeterminacy of a concept means that it cannot be clearly distinguished from other concepts. What, for example, are the boundaries of "life"? 
Up to what point are a pair of contested definitions incompatible, and when is the difference the origin of a discussion about definitions? In other words, a problem Gallie raises is that of the conflict of definitions, which in some cases can be the starting point for a new agreement on a univocal definition of the used term. In other cases it can cause a deadlock. In still others it can lead to a partial agreement that acknowledges the incompatibility of the opposed meanings and leads to a decision favouring the use of one of them.

\subsection{Emotive meaning and conflicts of values}

Stevenson $(1937$; 1944) noticed that words can have an emotive meaning, that is, they can lead the interlocutor towards effects different from cognitive effects attributable to the denotative meaning. The emotive meaning is defined in (Stevenson 1937, p. 23) as a tendency of a word to produce affective responses in people. This tendency is connected by Stevenson to the dynamic usage of a word, that is, the use of a term not to communicate beliefs, but to encourage in the interlocutor a feeling, an emotion, or to incite him to action. For instance, it is possible to refer to the same person, a 59 years-old lady, as an "elderly maid" or "old spinster". The last referring expression, we can notice, encourages the others to have contempt for the person so designated (p. 23). Stevenson thought that the main function of the word 'good', like the word 'hurray' is to encourage some type of behaviour in the interlocutor. He thought that saying something is good, like saying 'I like ice cream', is merely expressing a positive emotive feeling about the object.

On his view, ethical terms, such as 'good' or 'bad', the descriptive meaning cannot be separated from the emotive one. Such terms, on his view, have a more or less precise descriptive meaning, but at the same time they signify the approval or disapproval of the speaker and encourage an attitude in the interlocutor. For Stevenson, saying "this is a good thing" and "we all like this" are approximately the same (p. 25). While in some terms the emotive meaning is independent from the cognitive meaning, such as in the case of ethical terms, in other cases a word has an emotive meaning because its referent is positively evaluated. In other cases the relationship is more complex, such as in case of 'democracy', in which the descriptive meaning only partly determines the emotive one.

One of the most interesting aspects of Stevenson's theory is the distinction between disagreements about beliefs and disagreements about interests. A disagreement about interests can be manifested by expressions like "I like doing X" or by using ethical terms, such as in the sentence "Doing X is better". Disagreement about interests according to Stevenson, is in fact typical of ethics. However, they can also be rooted in disagreements about belief. On his view, incomplete knowledge or belief can be a cause of disagreements in interests, but the nature of these two types of conflicts is essentially different. 
This suggestion does not seem to be taken into consideration by any other theory on PDs. Schiappa and Burgess-Jackson treat the emotive aspect of PDs by inquiring into the relation between the common knowledge presupposed by the definition and the evaluation of it. Stevenson's account highlights two fundamental aspects of disagreements related to the emotional meaning: their connection with the concept of interest, and their relation to beliefs.

In the Nicomachean Ethics, the core of a decision is seen in its goal. The goal of the desire (or better, wish) can be what is good, or what appears to be good (III, 4, 1113a15); in fact, "everything aims at the good” (Topics III, 1, 116a 18). Moreover, the objects of choice are "the noble, the advantageous, the pleasant", while the objects of avoidance are "the base, the injurious, the painful". Pleasure, in particular, "accompanies all objects of choice; for even the noble and the advantageous appear pleasant” (Nicomachean Ethics, II, 3, 1104b 301105a 5). Happiness and goodness can be identified as the ultimate goals of actions, which directly or indirectly aim at them. This complex ethical theory can be simplified to the relation between evaluation, will, and choice. However, if we intend an action as related to a decision, connected to a goal and to a choice, the "emotive meaning" can be related to argumentation, and the "disagreements in intentions" to a set of reasons supporting a choice.

An intention, in our view, can be interpreted as a desire, a wish stemming from an evaluation, from what is or appears to be good. In this perspective, the notion of good is pivotal. While in the Nicomachean Ethics Aristotle defends an absolute perspective on goodness, related to the goal or function of life, in the Rhetoric and Topics he relates the concept of good to the reasons given . For instance, we can consider a list of topics which are useful to classify something as good (Rhetoric, I, VI):

The virtues, too, must be something good; for it is by possessing these that we are in a good condition, and they tend to produce good works and good actions. They must be severally named and described elsewhere. Pleasure, again, must be a good thing, since it is the nature of all animals to aim at it. Consequently both pleasant and beautiful things must be good things, since the former are productive of pleasure, while of the beautiful things some are pleasant and some desirable in and for themselves. The following is a more detailed list of things that must be good. Happiness, as being desirable in itself and sufficient by itself, and as being that for whose sake we choose many other things. Also justice, courage, temperance, magnanimity, magnificence, and all such qualities, as being excellences of the soul. Further, health, beauty, and the like, as being bodily excellences and productive of many other good things: for instance, health is productive both of pleasure and of life, and therefore is thought the greatest of goods, since these two things which it causes, pleasure and life, are two of the things most highly prized by ordinary people. 
In all these topics, we can notice, there is a reason given to support the classification. Aristotle, in the Topics, highlights another crucial aspectethical argumentation-that is, what Stevenson calls "conflict of interests". However, Aristotle recognizes that, along with an absolute concept of goodness, there is a relative one (Rhetoric I, 6; Topics I, 11, 115b, 22-26):

Further, a man of a given disposition makes chiefly for the corresponding things: lovers of victory make for victory, lovers of honour for honour, money-loving men for money, and so with the rest. These, then, are the sources from which we must derive our means of persuasion about Good and Utility.

In the same way also it is in certain places honourable to sacrifice one's father, e.g. among the Triballi, whereas, absolutely, it is not honourable. Or possibly this may indicate a relativity not to places but to persons: for it is all the same wherever they may be: for everywhere it will be held honourable among the Triballi themselves, just because they are Triballi. Again, at certain times it is a good thing to take medicines, e.g. when one is ill, but it is not so absolutely.

When to the interlocutors two things appear desirable in the same way, it is necessary to solve a kind of conflict of values. In the Topics and in the Rhetoric, Aristotle deals with conflicting values by formulating the topic of the preferable. For instance, a topic of this kind is (Topics, III 1, 2834):

That which is desired for itself is more desirable than that which is desired for something else; e.g. health is more desirable than gymnastics: for the former is desired for itself, the latter for something else. Also, that which is desirable in itself is more desirable than what is desirable per accidens; e.g. justice in our friends than justice in our enemies: for the former is desirable in itself, the latter per accidens: for we desire that our enemies should be just per accidens, in order that they may do us no harm.

Argumentation, in other words, can be used to solve disagreements about values. However, we should notice that there is an essential difference in arguing starting from a shared absolute notion of "good" or "goal of life", and conflicts involving the very concepts of happiness and goodness (see Nicomachean Ethics, I, 5). For some people the goal of life and happiness is pleasure, for others it is honour. There are levels of agreement and corresponding levels of conflict that can arise from values. This difference must be acknowledged, in order to recognize when a conflict of "interests" can be easily solved, or when the conflict becomes a conflict of different types of and perspectives on life and other philosophical matters. 
We conclude that in the so called "ethical meaning" two types of conflict can arise: conflicts stemming from the classification of something as good, and disagreements about what is better or worse. In both types of disputes, we should recognize that there might be different levels of disagreement, according to the different types of common ground.

\subsection{Conflicts of classifications and conflicts of values}

The purpose of the preceding subsection was to highlight the different types of conflicts and disagreements that can underlie persuasive definitions. Since PDs are redefinitions involving a persuasive effect, we inquired into the two aspects of this strategy using Stevenson's distinction. Conflicts can regard the classification of an aspect of reality, or its evaluation. Evaluation, in turn, can be basically conceived as a process of classification involving not definitions of (possibly) objective reality, but ethical values. In other words, conflicts can regard things or values, but both involve a disagreement about classification.

\section{Classification, decisions, and argument schemes}

In the previous section we identified different types and different levels of disagreement relative to PDs. At the first level, we recognized that we can find conflicts of classifications and conflicts regarding what we conceive as reasons leading somebody to act. We call this second type conflicts of values, meaning by 'value' what makes something desirable, and is therefore object of wish and, indirectly, action. At a second deeper level, we found that conflicts of values can be represented as conflicts of classification. The disagreement can be traced back to the reasons leading to the controversial evaluation of the situation. The conflict can be resolved by means of topics regarding the classification of something as "good" or "pleasant”, or "desirable”, grounded on the same definition of these concepts. Some disagreements, however, stem from different opinions about 'good'. Topics from the preferable are an instrument useful to decide which is better between two values.

In modern approaches to argument analysis, the theory of topics is preserved in what are called argumentation schemes. Argumentation schemes are abstract patterns of inference representing common forms of argument in everyday conversational reasoning and other contexts (Like law or science). They can be compared to the classifications by differentia in the medieval dialectical tradition (see Stump, 1984; GreenPedersen, 1989). Arguments from classification and values instantiate the complex system of Aristotelian "intrinsic" topics of definition, proper, genus, and accident (see Walton and Macagno 2007c). This type of argument can be formalized as follows (Walton 1996, p. 129): 


\section{Argument from verbal classification}

Individual Premise:

Classification Premise:

Conclusion: $a$ has property $F$.

For all $x$, if $x$ has property $F$, then $x$ can be classified as having property $G$.

$a$ has property $G$.

\section{Critical questions}

CQ1: What evidence is there that $a$ definitely has property $F$, as opposed to evidence indicating room for doubt on whether it should be so classified?

CQ2: Is the verbal classification in the classification premise based merely on an assumption about word usage that is subject to doubt?

Argument from classification is the instrument we can use to describe the phenomenon of conflicts of categorizations. Disagreements about values involve a more complex form of reasoning, linking evaluation to desirability and action. We can describe this process in a three-step reasoning:

(1) $x$ (an action, or an object, or a viewpoint) can be positively or negatively judged by agent $A$ according to a value $V$. For instance, stealing is evaluated negatively by agent Bob, because (for example) it is

(2) Depending on the desirability of $x, x$ can become a goal for the agent or not. For instance, because stealing is not desirable for Bob, stealing is not a goal for Bob.

(3) The fact that the action is or is not a goal determines whether the agent $A$ maintains or retracts his commitment to $x$. For instance, because stealing is not a goal for Bob, Bob does not commit himself to the action of stealing.

This type of reasoning can be applied both to action commitments and propositional commitments. We represent the scheme as follows (Walton, Reed and Macagno, 2008, Chapter 9):

\section{Argument from values Variant 1: Positive value}

Premise 1: $\quad$ Value $V$ is positive as judged by agent $A$ (judgment value).

Premise 2: $\quad$ The fact that value $V$ is positive affects the interpretation and therefore the evaluation of goal $G$ of agent $A$. (If value $V$ is good, it supports commitment to goal $G$ ).

Conclusion: $\quad V$ is a reason for retaining commitment to goal $G$. 


\section{Argument from values Variant 2: Negative value}

Premise 1: $\quad$ Value $V$ is negative as judged by agent $A$ (judgment value).

Premise 2: $\quad$ The fact that value $V$ is negative affects the interpretation and therefore the evaluation of goal $G$ of agent $A$. (If value $V$ is bad, it goes against commitment to goal $G$.)

Conclusion: $\quad V$ is a reason for retracting commitment to goal $G$.

The argument schemes from classification and values are the tools that can be used to analyse the types of conflicts involved in persuasive definitions. Presenting this analysis will be the object of the following sections.

\section{PDs: Persuasiveness in the definiendum, in the definiens, and complex cases}

A definition involves both something that is defined and a discourse that explains its meaning (Aristotle, Topics I, 5, 102 a 1-2). In order to understand the disagreements that persuasive definition is based on, we classify the two categories of what Stevenson called Persuasive Definitions and Quasi-Definitions (respectively) as strategies focused on the definiens, and strategies centred on the definiendum. While in the first case the persuasive effect is grounded on a form of ambiguity of the definiendum, stemming from the polisemy introduced by means of a redefinition, in the second case it is the implicit argumentation from values (the emotive meaning) that is object of the argumentative manoeuvre.

\subsection{Redefinitions and implicit conflicts of meaning}

Persuasive definition involving a redefinition of a concept trades on the ambiguity of meaning and the implicit argumentation from values associated to the original meaning of the term. We want in this subsection analyze that strategy by showing the reasoning patterns involved, and suggesting how it can be explained.

Regarding the first point, we start from an example. Stevenson (1944, p. 211) offered the following example in the form of a dialogue.

\section{Case 1. The dialogue on culture}

A: He has had but little formal education, as is plainly evident from his conversation. His sentences are often roughly cast, his historical and literary references rather obvious, and his thinking is wanting in that subtlety and sophistication which mark a trained intellect. He is definitely lacking in culture.

B: Much of what you say is true, but I should call him a man of culture notwithstanding. 
A: Aren't the characteristics I mention the antithesis of culture, contrary to the very meaning, of the term?

B: By no means. You are stressing the outward forms, simply the empty shell of culture. In the true and full sense of the term, "culture" means imaginative sensitivity and originality. These qualities he has; and so I say, and indeed with no little humility, that he is a man of far deeper culture than many of us who have had superior advantages in education.

Using this case an example, we represent the strategy of persuasive definition as a conflict between the implicit conclusions of two implicit arguments from values. Persuasive definition trades on the same values, or better, reasons for action, of the interlocutor, which we represent as a double ownership given to the premise 'Culture is a positive quality' in Figure 1. The manoeuvre, however, is grounded on an ambiguity of meaning, which we represent as a double arrow in Figure1. The interlocutors, while founding their arguments from values on the same value, are grounding them on two different classifications, covered by the same name. Using the traditional terminology, A can be said to be using a quaternio terminorum, because he introduces a polisemy that is later exploited in the implicit argument leading to a commitment.

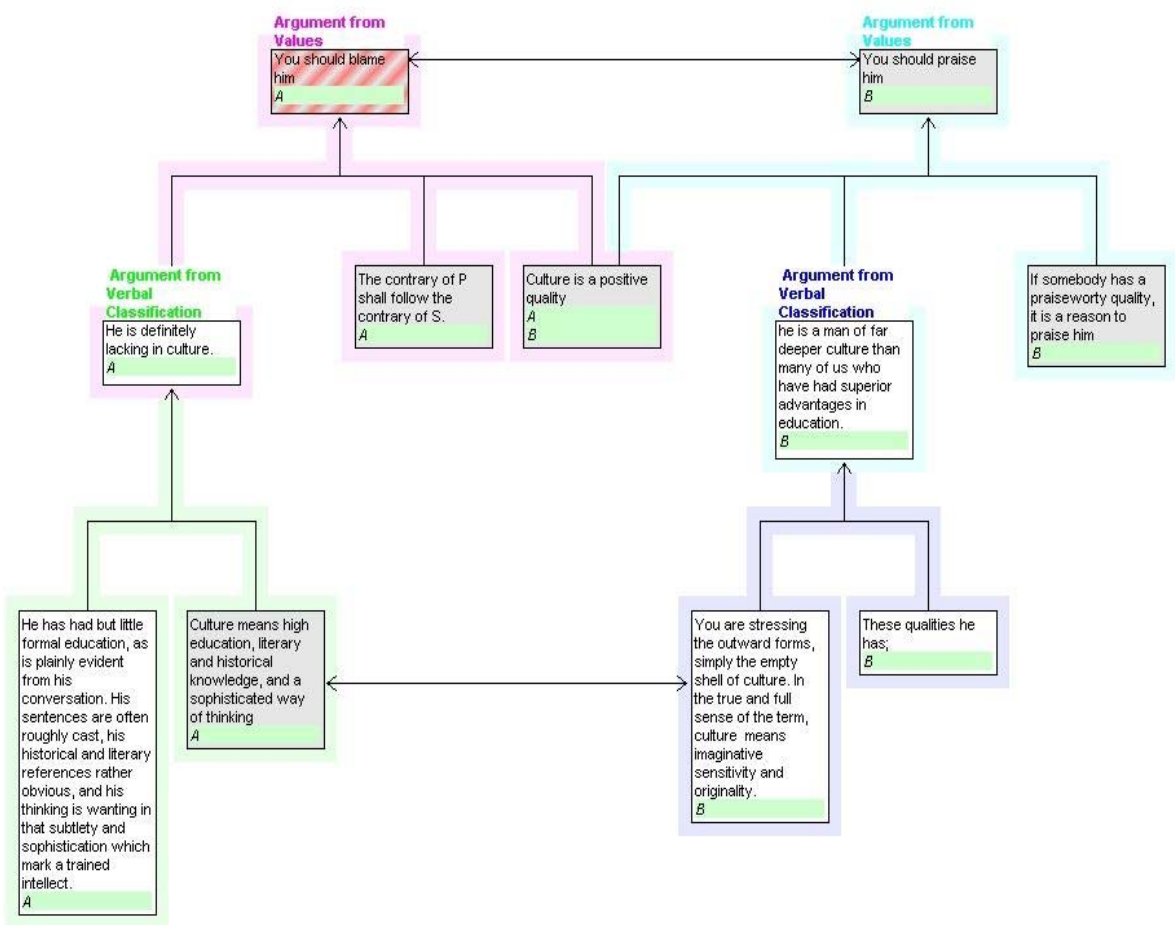

Figure 1. Conflicts of classifications

The form of the culture example can be found in many similar cases of redefinition in ordinary discourse. For instance, consider the following example: 


\section{Fabrizio Macagno \& Douglas Walton}

\section{Case 2. Redefinition of taxation}

Libertarians will define "taxation” as "a form of theft," thus making supporters of at least some sort of government taxation look like thieves, not to mention the government itself.

To deal with cases like 1 and 2 it is useful to recognize the introduced polisemy and submit the argumentation to a clarification dialogue. The distinction of meaning is extremely useful for disagreements that apparently are about values, but in fact stem from a misunderstanding. This theme can be better explained by means of a case of apparently conflicting definitions. We show in the example in Case 3 below how a distinction of meaning is fundamental to avoid a deadlock in an apparent conflict of values (Walton 2006, pp. 37-38):

\section{Case 3. Euthanasia dialogue}

The issue of a debate between two people was whether or not euthanasia should be legalized. One party (A) argues that it is morally justified, and should therefore be legalized, because it offers terminally ill patients who are enduring unbearable suffering an opportunity to die with dignity. She argues that such a patient should be able, on request, to be taken off lifesupport systems when aggressive or heroic treatments are no longer doing any good to save her life. The other party (B) disagrees, arguing that euthanasia is murder and that any physician who gives a patient a lethal drug is killing that person, even if the patient agrees to it. The one (B) party keeps insisting that euthanasia is murder, while the other $(\mathrm{A})$ denies that it is murder at all, saying that it is 'letting nature take its course", without high-tech interference.

Here the disagreement stems from two different definitions of euthanasia, from which a disagreement in values emerges. The problem is that the two people (whom we call A and B) are not talking about the same thing. $A$ is referring to passive euthanasia, while $B$ to active euthanasia. The argument from values overlaps the argument from classification, and resolving the problem of classification means partly avoiding the deadlock of contrasting argumentation from values. 


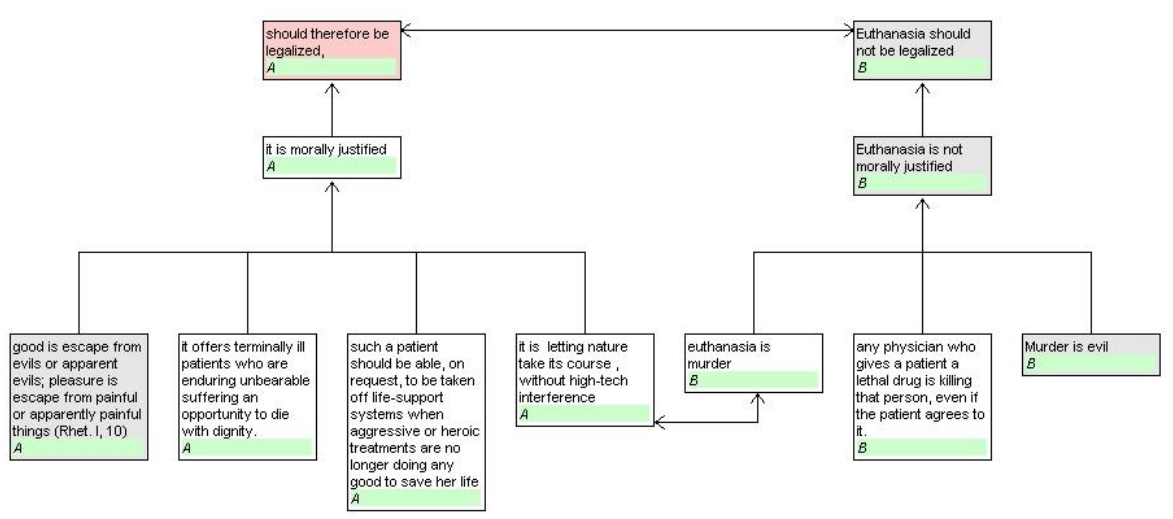

Figure 2. Apparent conflict of definitions

The device of submitting to a clarification dialogue can be useful in such a case if the interlocutors have at least a basis of agreement. The redefinition can, in fact, generate cases of deadlock. Price (2001) described how the phenomenon of PD's gave rise to a situation of failure of communication between the ancient Greek republics. Concepts such as 'freedom' were persuasively redefined, but the conflict generated from it prevented mutual understanding.

Clarification dialogue can be used when a conflict of meaning is individuated. The subtlest strategy consists of using a word that presupposes a redefinition. The original meaning of term is changed, in order to predicate it of a fragment of reality it otherwise would not apply to. However, the definition is not stated explicitly. This technique trades both on ambiguity and manipulation of the common ground. For instance, in the case below, the term 'satisfied society' has been implicitly redefined (Capria 1999, p. 3):

\section{Case 4. The satisfied society}

In a book of his from 1968, used as a text at Yale for many years, Huntington classifies South Africa as a "society with low systemic frustration" (referring to the definition of "frustration ratio" given by I.K. and R.L. Feierabend in an article from 1966), and so no less than a "satisfied society". (Note that at that time, South Africa was at the height of apartheid, with 20 million Blacks victims of racial segregation.) Lang has a field day in showing the pseudoscientific and intimidatory nature of the equations included in Huntington's book (involving "quantities" such as "social mobilization", "political institutionalization" and the like), and the silence on riots and repression, which were mentioned even in newspapers in the decade preceding the book's publication. Huntington does not answer directly, but in a magazine interview, he assertswithout any concern for the "frustration ratio"—-that a "satisfied society" has for him the following peculiar technical meaning: 


\section{Fabrizio Macagno \& Douglas Walton}

whether the lot of the people is "good, fair, or awful [...] the people for some reasons are not protesting it."

Disagreement about meaning is, we conclude, often at the basis of a disagreement about values. While in disagreements about definitions the ambiguity of a term is an involuntary hindrance for the goal of the dialogue, in PD's this phenomenon is used to support an argument from values. This possible solution to the problem of redefinitions allows us to understand the most common way they are used, that is, implicitly. Terms are simply used with a new definition. In this way, the interlocutor cannot individuate a disagreement in meaning. What we called disagreement in meaning represents Stevenson's persuasive definition. The counterpart of this manoeuvre is the quasi-definition, or, in our view, disagreements about values.

\subsection{Quasi-definitions and conflicts of values}

As mentioned above, quasi-definitions can be described as phenomena of redefinition involving a particular implicit argumentation from values. The speaker advances his argumentation supporting his conclusion by means of values contrasting, or partly conflicting, with the interlocutor's one. We can better explain this strategy with the following example (Molière, 2000, pp. 98-99):

\section{Case 5. Marriage and values}

DON JUAN: What! Would you restrict a man to staying chained to the first woman who takes his fancy, have him give up everything for her and never look at any others again? The idea is ludicrous - making a bogus virtue out of being faithful, being trapped forever in the same relationship and as good as dead from youth onwards to the other pretty faces that might catch our eye! No no: fidelity is for imbeciles. All beautiful women are entitled to our love, and the accident of being the first on the scene shouldn't deprive the rest of the rightful claims they have on our affections. Speaking for myself, beauty enchants me wherever I find it and I surrender unresistingly to the sweet savagery it stirs in us. No matter how far I'm committed, the fact that I'm in love with one beautiful woman shall never make me unfair to the rest.

Here we should notice that word 'marriage' maintains its descriptive meaning, while it is the emotive meaning that has been altered. In other words, we can describe the strategy as centred on argument from values, instead of argument from classification. The value of fidelity is contrasted with fairness. We can represent the pattern of reasoning as shown in Figure 3: 


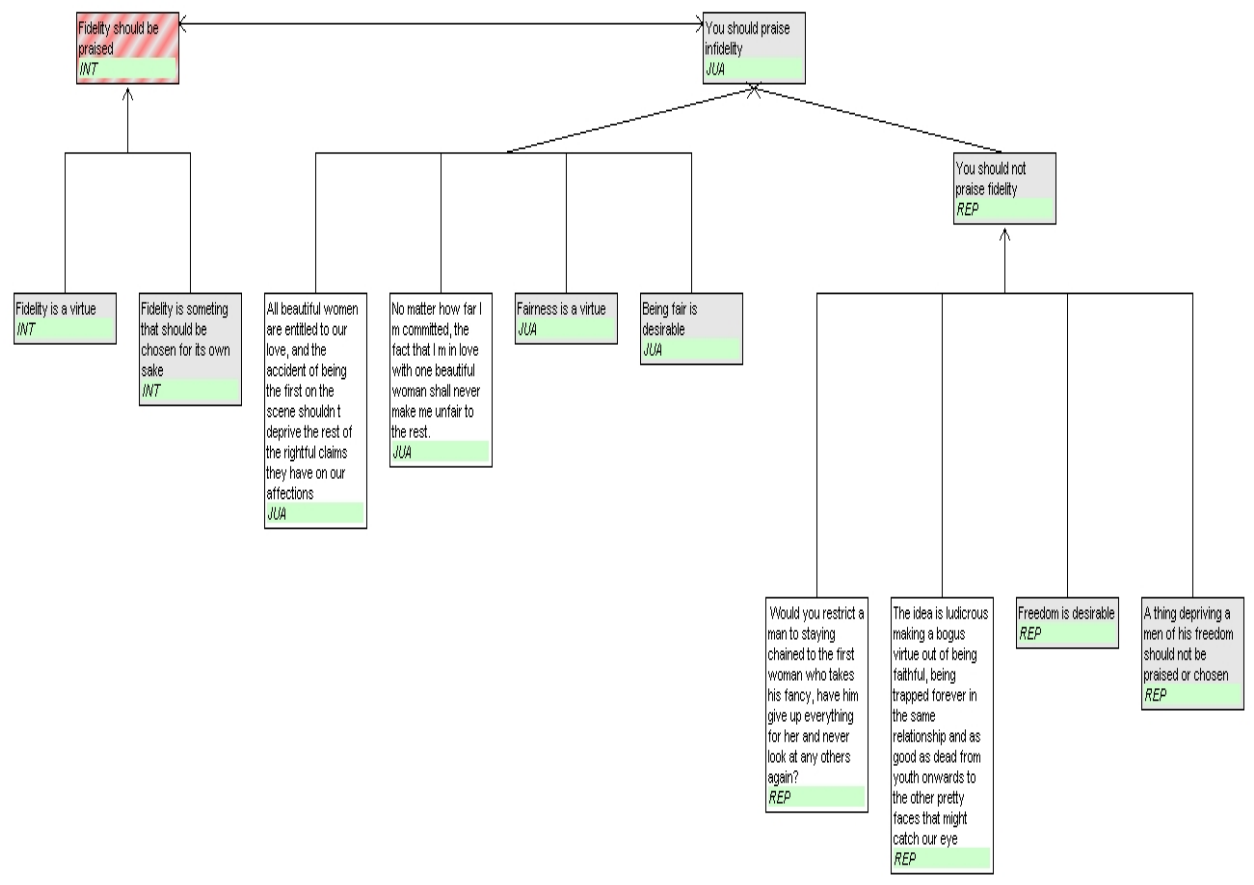

Figure 3. Marriage example

Here the strategy stems from a representation (REP) of the ordinary concept of being faithful. Don Juan highlights the fact that fidelity implies loss of freedom, and opposes loss of freedom to a redefined concept of fairness. We need to notice that this is a conflict of values, since the original meaning of 'marriage' is preserved. However, a complex implicit line of argumentation supports the view that infidelity is desirable. It can be examined at different levels.

At a first level, there is a conflict between "fidelity" and "freedom" and "fairness". The second values are presented as more desirable than the first. We could represent this comparison by means of argumentation from the better. Even tough the interlocutor (INT) accepts the implicit redefinition of "fairness" and "freedom", she would hardly recognize that these values are superior to virtue.

At a second level, this quasi-definition is grounded upon a persuasive definition of "fairness", "freedom", and "fidelity". The conflict of values can, in this perspective, be solved at the level of conflict of meaning. The two levels are represented in Figure 4. 


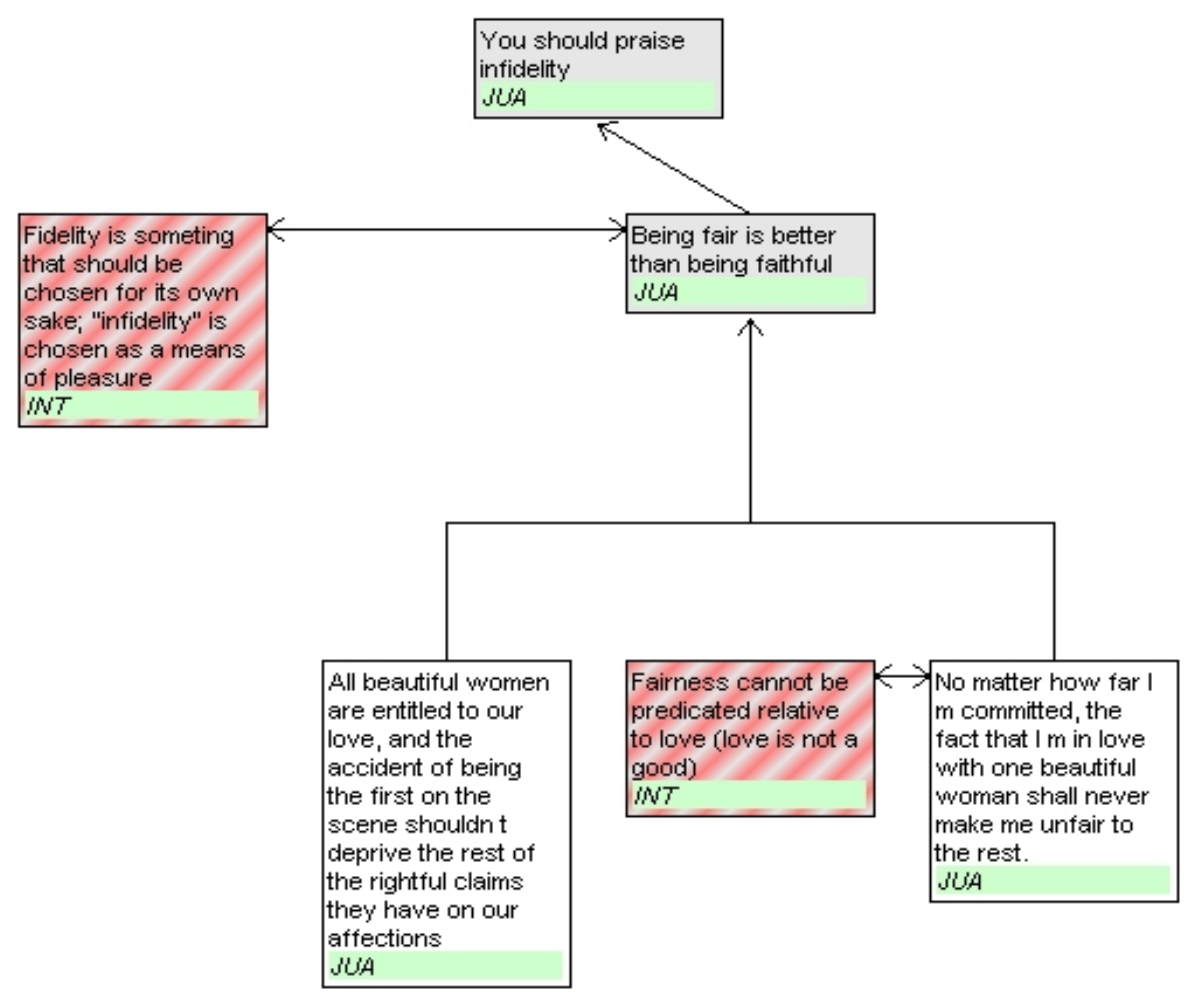

Figure 4. Conflicts of Values and Conflicts of meaning

The conflict about the definition of virtues and values is crucial in PD's. This conflict underlies the most common argumentation strategies associated with PD's, and can be solved by means of embedding a clarification dialogue into the ongoing argumentation. We can illustrate how an implicit redefinition of values can be used to support an argument, and how a clarification dialogue can locate the source of the disagreement. To do this, we use Case 6 (Ennis, 1996, pp. 252-253).

\section{Case 6. The dialogue on cutting in line}

JOHN: You were selfish in cutting into that lunch line when those people weren't looking.

MARY: So what? I'd just did what anyone would do.

JOHN: That's not true, but it doesn't matter whether other people would do that. It was selfish.

MARY: Let's define our terms. To be selfish is to act in accord with your very own desires. Right?

JOHN: Hmm. I'm not sure.

MARY: That's an enlightened definition of the word selfish. It makes deciding about whether someone is selfish a purely factual matter. People have desires. If they act in accord with them, then they're selfish. It's as simple as that. This definition leaves out the subjective meaning that some people have for the word selfish, so it is a better definition. 
JOHN: I follow you so far. Go on.

MARY: Good. Now you should be able to see that everything everybody does is really selfish. That's because people really act in accord with their desires all the time. If they did not desire to do with what they do, they would not do it.

JOHN: So?

MARY: Because everything everybody does is selfish, you cannot condemn me for doing something selfish. That wouldn't be fair.

In his commentary on the cutting in line example, Ennis (1996, p. 353) observed that Mary has managed to make it appear that her act of cutting in line was not a bad thing, or at any rate is no worse than anything done by anyone else. The reason is that according to Mary's redefinition of the word selfish, together with the premise that people always act in accord with their desires, it follows that being selfish is unavoidable, and therefore cannot be wrong, or subject to moral condemnation.

Ennis (p. 353) pointed out that there are several different kinds of responses that John could make to Mary's argument. Let's consider two of them. One response is for John to accept Mary's definition and argument, but then to claim that Mary's cutting into line was still unfair to the people who were ahead of her in the line. Another response is for John to distinguish different types of selfishness. He can argue that there is good selfishness, bad selfishness, and a third kind of selfishness that is neither good nor bad. From this vantage point he could argue that Mary's cutting into line should be classified into the category of the bad type of selfishness.

\subsection{Complex cases}

The two categories distinguished by Stevenson, PD's and quasidefinitions, are often mixed. There can be a redefinition of a concept together with an implicit sequence of reasoning aimed at altering the argumentation from values commonly associated with the concept. We analyze the following example, one that shows how persuasive definitions are used in advertisements (Aberdein, 2000, p. 1).

\section{Case 7. The temperance example}

And the brewers' trade papers: they're full of articles about the beauty of true temperance. Ordinary temperance is just gross refusal to drink; but true temperance is something much more refined. True temperance is a bottle of claret with each meal and three double whiskies after dinner.

Here the redefinition which introduces the polisemy of the virtue 'temperance' is introduced by use of the word 'true'. The implicit conclusion would be a commitment to the view that consumption of alcohol should be seen as acceptable. However, we notice in this case 
that the ordinary meaning of 'temperance' is not even taken into consideration. The term is redefined in two distinct ways, evaluating each of them by means of the adjectives 'gross' and 'refined'. Argumentation from values is mixed in with the redefinition. The argument is trading on both the ambiguity of the term 'temperance' and the evaluation of the concept of "a bottle of claret with each meal" as something "refined". Our analysis of this case is shown in Figure 5.

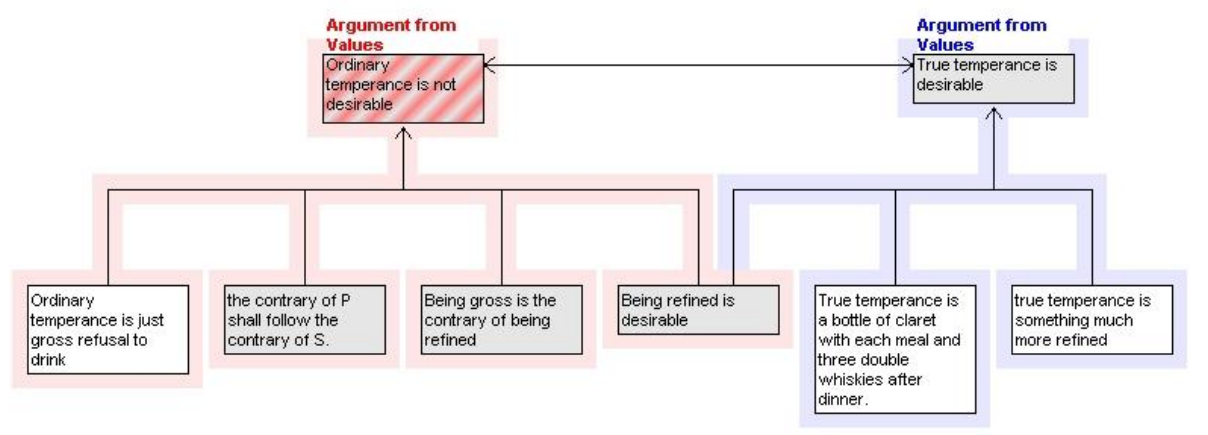

Figure 5. Complex cases: The temperance example

As the argumentation is shown in Figure 5, this is not a case of simple quasi-definition, nor one of persuasive-definition. It is a complex mix of both of them. Two new definitions of 'temperance' are introduced, conflicting with the way the word is ordinary used, and the evaluation of 'temperance' from the point of view of virtue (that is, using a line of argument stemming from the concept of virtue) is replaced by the assessment using the values "prestige" and "quality of being refined". The deeper level of conflicts can be represented in Figure 6, below.

We should observe in Figure 6 how the implicit disagreements (marked by a double line and a double arrow) involve both a conflict of meaning and a conflict of values. A black or white fallacy can be seen in the dichotomy between "ordinary temperance" and "true temperance", while the rest of the strategy is grounded upon the implicit value "prestige". If we want to analyze this case even more deeply, we can say that it involves a manipulation of values. Temperance is presented as desirable not per se, as a virtue, but because it is a way of being refined.

A similar case is the following, in which first the meaning of "heathen" is changed from the ordinary use of it, and then the value of "faith" is altered, presented as something ridiculous. 


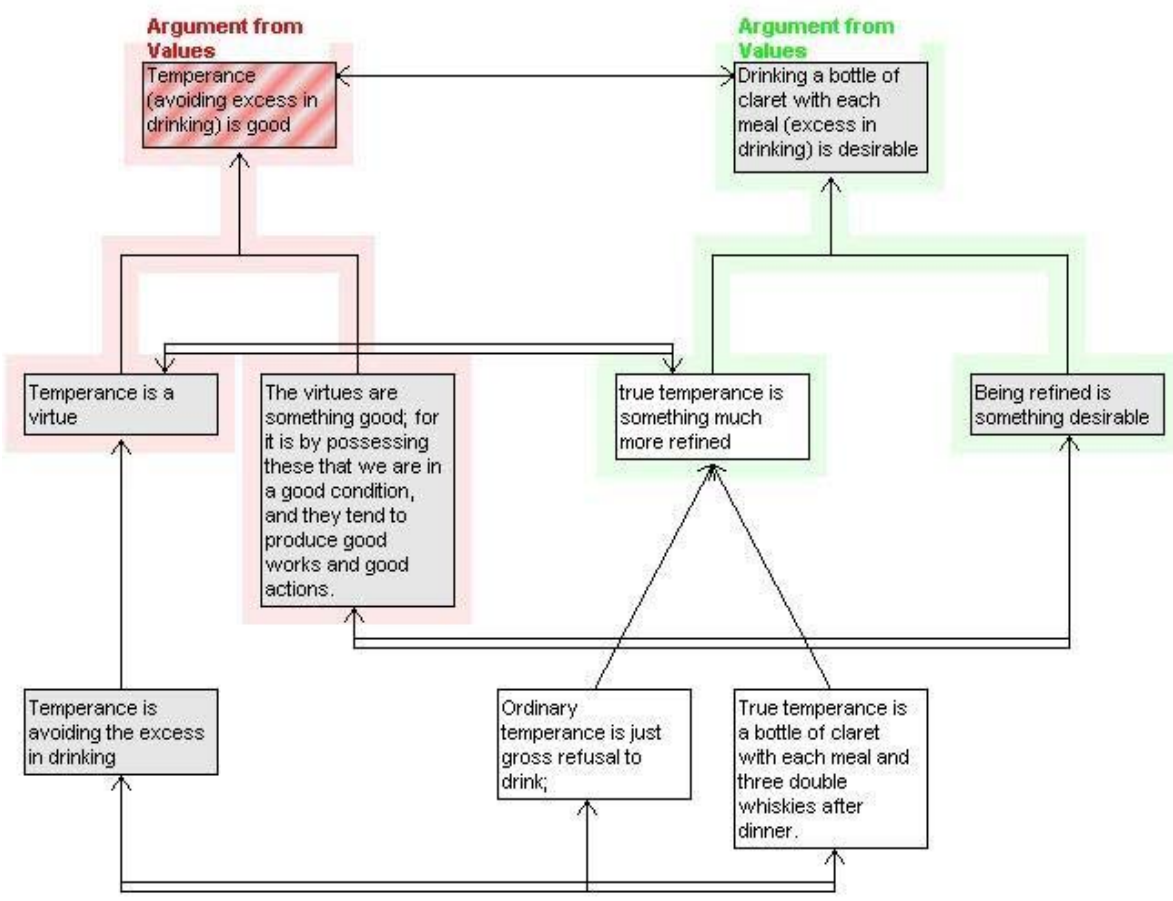

Figure 6. Types of disagreement in complex cases

\section{Case 8. The heathen example}

For example, Ambrose Bierce, in The Devil's Dictionary, defines "heathen" as "a benighted creature who has the folly to worship something that he can see and feel." The lexical definition would usually be along the lines of "one who does not believe in the Christian God" or "one who does not follow an established religion. Bierce's definition attempts to make the case for the heathen's position (by using irony), suggesting that it is foolish to believe in something you cannot see or feel. This definition would be inappropriate for theological argument, as it is biased toward one side.

Complex cases, to conclude, involve both a PD and a quasi-definition, which have different and interrelated goals and functions.

\section{Conflicts of meanings, conflicts of values, and communication failure}

In the subsections above we explained PDs in terms of disagreements about meaning and about values. While disagreements about meanings can be resolved with clarification dialogues, we analyzed conflicts of values using the argumentation from "the better" and clarification dialogue about the meaning of values. We showed how many conflicts about values can be disagreements about the meaning of values. In this 
section, we will focus on the two aspects of argumentation from values, that is, on dialogues about what is better, and dialogues about the meaning of a value. These two discussions are placed on different levels. In order for the interlocutors to discuss about what is better, they have to share a common meaning of 'better' and of the values they are discussing. The clarification dialogue is at a deeper level, when there is not a common ground for solving the disagreement at a dialogical level. The interlocutors have to shift the dialogue to a kind of metadialogue, that is, a secondary dialogue at a higher level about a prior dialogue already taking place at a primary level (Krabbe, 2003). When a misunderstanding or procedural disagreement prevents a primary dialogue from moving forward, the problem needs to be solved by moving to a clarification dialogue, that takes place at a metadialogue level.

\subsection{Dialogues about "what is better" and clarification metadialogues}

In order to show how a conflict of values can hide different levels of disagreement, it is useful to analyze one of the most controversial contemporary issues, the debate about abortion. Starting from two different definitions of 'abortion', that could be summarized as 'murder' and 'a woman's choice of controlling her own body', we can highlight the type of conflict and how it can be dealt with.

\section{Case 9. Life versus choice}

Although some oppose abortion for being used as a form of birth control, Abortion should remain legal because it is a woman's choice and protects the privacy of an individual. [...] Abortion is a woman's choice. Women accepted the definition that a woman's prime role was as wife and mother and control of ones own body. Once they had choices about life roles, they came to feel they had the right to choose abortion to run their own lives. Any woman should have the ability to choose when to have a child in their marital and sexual freedom. Many women feel if they didn't have the right to choose an abortion in their life they would have passed by many opportunities to create a better life for themselves .

Regardless of the fact of morals, a woman has the right to privacy and her choice to abort. Only the woman has the control over her body and reproductive system. I believe abortion is a woman's choice because I feel that a woman has the right to decide what is right or wrong for her, her body, and her life.

A pro-choice person would feel that the decision to abort a pregnancy is that of the mother and the government has no right to interfere. A pro-life person would believe that from the moment of conception, the embryo or fetus is alive. Since this embryo or fetus is alive and is a person, you have no moral right 
to abort a life. If you aborted the life (person) you would be committing murder.

In this example, we should notice that there is a conflict of values: life versus freedom. This situation, however, is highly complex. We could represent it at a first level of conflict of values as shown in Figure 7.

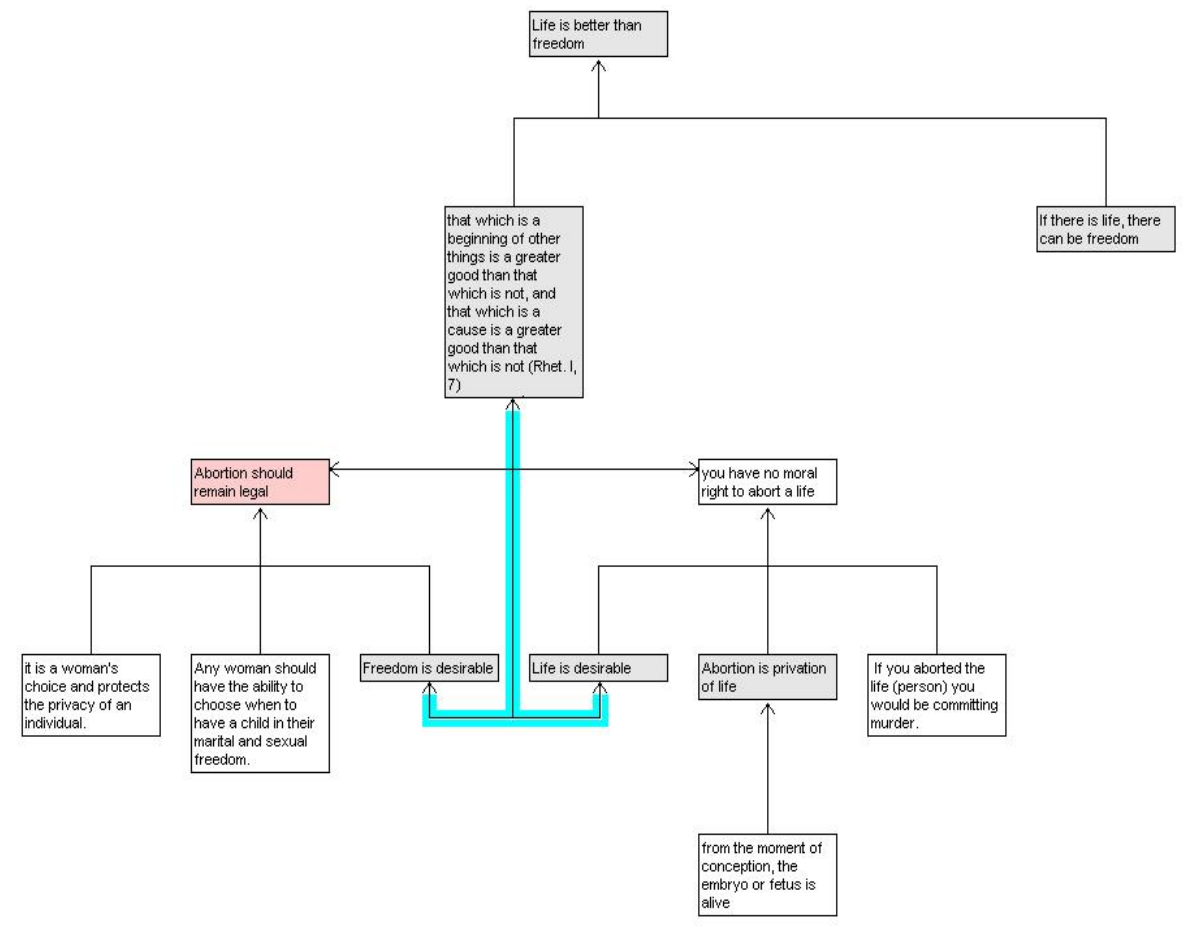

Figure 7. Argumentation from preferable

In the diagram shown in Figure 7, we need to notice that the disagreement about values leads to possible argumentation from what is taken to be preferable. In this example we have chosen only one possible type of argument of this sort, considering the fact that the interlocutors share the same ideal of life and freedom. However, we should notice that in Case 9 it is the very concept of life that is at stake. The argument from what is preferable would not be acceptable for a pro-choice advocate, since usually positions of this type are grounded on a definition of fetus or embryo different from that of the pro-life advocate. In order to resolve this type of dispute, it is therefore necessary to shift to a clarification dialogue about how 'fetus' should be defined, and, even more deeply, how 'life' is to be defined. The clarification dialogue between the two opposed sides concerning such an issue, however, can hardly be expected to lead to a successful result. The concept of life is too vague, too ambiguous, and to open to dispute itself. The deadlocked clarification dialogue prevents the primary dialogue from moving forward to resolution. 


\title{
4.2 Clarification dialogues and communication failure
}

In the case above, we observed how a conflict of values, in order to be solved, presupposes shared concepts of what the conflicting values are. We noticed, moreover, how in some cases a conflict of opinions regarding the meaning of a term implies a much deeper disagreement. In order for a clarification dialogue to be successful, the interlocutors must in fact have a common understanding of what a definition is, and what a better definition is. Aristotle, in the Topics, illustrates different types of rules concerning the object of a definition, the characteristics it has to fulfil, and the procedural rules of dialogue that have to be respected. For instance, we need to consider two principles (Topics 110a, 16-22; 151b 6-11; see also Giuliani, 1972, p. 130; 134)

\begin{abstract}
Moreover, you should define what kind of things should be called as most men call them, and what should not. For this is useful both for establishing and for overthrowing a view: e.g. you should say that we ought to use our terms to mean the same things as most people mean by them, but when we ask what kind of things are or are not of such and such a kind, we should not here go with the multitude: e.g. it is right to call 'healthy' whatever tends to produce health, as do most men: but in saying whether the object before us tends to produce health or not, we should adopt the language no longer of the multitude but of the doctor.
\end{abstract}

For the answerer is bound either to accept the sense as taken by the questioner, or else himself to explain clearly whatever it is that his definition means. Moreover, just as in the assemblies the ordinary practice is to move an emendation of the existing law and, if the emendation is better, they repeal the existing law, so one ought to do in the case of definitions as well: one ought oneself to propose a second definition: for if it is seen to be better, and more indicative of the object defined, clearly the definition already laid down will have been demolished, on the principle that there cannot be more than one definition of the same thing.

These types of rules are examples of the simplest forms of agreement necessary for a dialogue to be successful. When the object and the norms regulating a dialogue are not shared, a deadlock is inevitable. In order to explain this level of disagreement it can be useful to start with the analysis of the example in Case 10.

\section{Case 10. Dialogue about ethics}

B: [...] I must say that the deviations from the more fundamental and true moral ideals are simply perversions and corruptions. Whoever denies the principles of justice and neighborliness is immoral. [...] The ideals that I enumerated are the very essence of what is meant by "morality." To be 
moral consists precisely in placing oneself in the service of interests and ideals that transcend purely selfish purposes.

A: This is what you mean by 'morality.' (And, of course, it is in keeping with traditional morality). But Nietzsche, for example, explicitly proposed a revolution in all traditional morality. Clearly, he considered his own value-system as the "true ethics." Are you not aware that you are begging the very question at issue? You speak of "true moral ideals"; you call certain views "immoral," "perverse," "corrupt"; you say that only certain types of principles can "justifiably be called ethical." You are using persuasive definitions here. You call "moral" or "ethical" only such doctrines or principles as agree with your own convictions about what is right. [...] You cannot by some verbal magic establish justifications for ideals which only obviously are neither logically nor empirically unique. These ideals compete with genuine alternatives.

B: I can't believe this. The ideals that I have listed are the ones that will benefit humanity in the long run. Not just a particular group, but all of mankind. Moreover these ideals are comprised by the essence of rationality. Man, the rational animal, is by his very nature not only characterized by his capacity for adequate deductive and inductive thinking, but also by his sense of justice and his abhorrence of violence as a method for the settlement of disputes.

A: You are still begging the question. Those who do not accept the principle of equality are not interested in all of mankind. Furthermore, your time-honored conception of human nature is clearly not an account of actual fact, but of an ideal (by no means universally shared) which you utilize for a persuasive definition of MAN. You won't convince any serious opponents by mere definitions. [...].(Feigl, 1952)

This discussion can be described as a clarification dialogue about what morality and ethics are, stemming from an argument about values. However, the dialogue shifts to a metadialogue about how to define a term. B defines 'morality' using the common way this term is used, but A, after disagreeing about the way the term has been paraphrased, objects the very mode of the definition. He does not accept that the way a term is commonly used should be the mode of definition. $\mathrm{B}$ tries to anchor the definition to a common understating of what is 'man', but this leads to a further disagreement about what the essence of man is, and about which principles should be considered to be the basis of common knowledge.

In this case, we should observe, there is a deadlock, since the procedural rules and fundamental principles of a discussion are not shared. A's position is destructive, preventing any kind of possible agreement from being reached. Moreover, the mode of definition required is not common to the interlocutors. In this case we can talk about a secondary disagreement about dialogical principles. 


\section{Conclusion}

In this paper we analyzed the different types of disputes that can arise from persuasive definitions. Persuasive definitions are strategies of redefinition involving an implicit use of argument from values. PDs are commonly recognized as fallacious: a definition is usually condemned as persuasive when it is seen as a deceptive strategy used to manipulate the other party. Such manipulation presupposes a potential conflict of opinions or perspectives. In this paper we presented a solution to the problem of revealing the implicit conflicts presupposed by the use of a PD. The explanation we provided, by distinguishing between different types and levels of disagreements, led us to solve two related problems, namely how these different types of conflicts can be resolved, and how differences of opinion can cause a deadlock.

After introducing the notion of persuasive definition and explaining how such definitions work in argumentation, we centred our study on the two types of meaning that Stevenson takes into consideration, namely descriptive and emotive meaning. We examined the most influential theories about PD in order to provide a background for our approach and show how it represents an improvement. The previous studies of the argumentative use of definitions offer interesting viewpoints that attempt to deal with the problem of disagreements. These approaches highlight the crucial function of common ground, values, and polisemy in PDs. These notions also constitute the basis of our account. However, instead of separating the two types of meaning, we showed how they can be integrated in an argumentation approach to PD. Starting from an Aristotelian perspective on action and decision, we interpreted emotive meaning as an implicit use of argument from values. This type of argument can be part of the attempt to resolve disagreement at the level of the values involved, and at the level of the meaning of values.

The second section of the paper was aimed at presenting the tools we used in our analysis, and our general approach to the problem of PD. Argument from classification and argument from values are used to describe the different levels of disagreement, as shown in the third section, and applied to studying the types of disagreement. As we showed, persuasive definitions and quasi-definitions trade on different strategies of persuasion, each associated with a distinct type of disagreement. While the former exploit the ambiguity of the definiendum, the latter change the assessment of a concept by advancing in the definiens a new evaluative viewpoint on the reality it refers to. In terms of disagreements, the former hide a disagreement about the meaning of the definiendum, the latter a disagreement about values and meaning of values. These types of implicit conflicts can furthermore be subdivided in levels of seriousness according to their relations to the common ground.

In the last section we explained how the levels of value disagreement can be dealt with by shifting the dialogue to distinct kinds of metadialogues, such as a clarification dialogue about a key definition. The nature and level of the disagreement, in such cases, can lead to 
different type of shifts or communicative failures when the most fundamental endoxa and the rules of dialogue are not shared by the parties to the dialogue.

\section{References}

Aberdein, Andrew. (2000). "Persuasive Definition." In Christopher W. Tindale, Hans V. Hansen and Elmar Sveda (eds.). Argumentation at the Century's Turn, OSSA (Ontario Society for the Study of Argumentation) Proceedings. CD ROM.

Aristotle. (1851). On Rhetoric. Translated by T. Buckley. London: Henry G. Bohn.

Aristotle. (1939). Topica. Translated by E.S. Forster, Loeb Classical Library. Cambridge, MA: Harvard University Press.

Aristotle. (1985). Nicomachean Ethics. Translated by Terence Irwin. Indianapolis, IN: Hackett.

Burgess-Jackson, K. (1995). "Rape and Persuasive Definition.” Canadian Journal of Philosophy 25: 415-454.

Capria, Marco Mamone. (1999). "Questions of Scientific Responsibility."

Review of Serge Lang, Challenges, Springer Verlag, New York 1998, pp. x+816. Review originally published in Punti Critici I/ 2, (September/December 1999). Unpublished English translation by Filippo Naitana, approved by the author.

Clarke, Barry. (1979). "Eccentrically Contested Concepts." British Journal of Political Science 9/1: 122-126.

Ennis, Robert. (1996). Critical Thinking. Upper Saddle River, NJ: Prentice-Hall.

Feigl, Herbert. (1952). "Validation and Vindication: An Analysis of the Nature and the Limits of Ethical Arguments." In Wilfrid Sellars and John Hospers (Eds.), Readings in Ethical Theory, pp. 667-680. New York: Appleton-Century-Crofts.

Gallie, Walter Bryce. (1956). "Essentially Contested Concepts." Proceedings of the Aristotelian Society 56: 167-198

Giuliani, Alessandro. (1972). "The Aristotelian Theory of the Dialectical Definition." Philosophy \& Rhetoric 5: 129-142

Green-Pedersen, Niels J. (1984). The Tradition of Topics in the Middle Age. Munich: Philosophia Verlag.

Hallden, Soren. (1960). True Love, True Humour and True Religion: A Semantic Study. Lund: Gleerlup.

Krabbe, Erik C.W. (2003). "Metadialogues." In Frans H. van Eemeren, J. Anthony Blair, Charles A. Willard and A. Francisca Snoeck Henkemans (eds.), Anyone Who Has a View: Theoretical Contributions to the Study of Argumentation, pp. 83-90. Dordrecht: Kluwer.

Molière, Jean-Baptiste. (2000). “Don Juan.” In J. Wood \& D. Coward (trans.), Molière. The Miser and other plays. Toronto: Penguin Books. 
Price, Jonathan. (2001). Thucydides and Internal war. Cambridge: Cambridge University Press.

Rigotti, Eddo. (2005). "Congruity Theory and Argumentation." Studies in Communication Sciences: 75-96.

Schiappa, Edward. (2003). Defining Reality: Definitions and the Politics of Meaning. Carbondale, IL: Southern Illinois University Press.

Stevenson, Charles L. (1937). "The Emotive Meaning of Ethical Terms." Mind 46: 14-31.

Stevenson, Charles L. (1938). "Persuasive Definitions." Mind 47: 331350.

Stevenson, Charles L. (1944). Ethics and Language. New Haven: Yale University Press.

Stump, Eleanore (1989). Dialectic and its place in the development of medieval logic. Ithaca, NY: Cornell University Press.

Vendler, Zeno (1964). "The Grammar of Goodness." The Philosophical Review 72 (4): 446-465.

Walton, Douglas \& Macagno Fabrizio (2007a, to appear). "Rhetorical Argumentation using Emotive Words.”

Walton, Douglas \& Macagno Fabrizio . "Reasoning from Classifications and Definitions”, Argumentation, 2007b: http://dx.doi.org/10.1007/s10503-008-9110-2

Macagno, Fabrizio \& Walton, Douglas, "The Argumentative Structure of Persuasive Definitions”, Ethical Theory and Moral Practice, 2007c: http://dx.doi.org/10.1007/s10677-008-9119-5

Walton, Douglas N. \& Krabbe, Erik C.W. (1995). Commitment in Dialogue. Albany, N.Y.: State University of New York Press.

Walton, Douglas (1996). Argumentation Schemes for Presumptive Reasoning. Mahwah, N.J.: Lawrence Erlbaum Publishers.

Walton, Douglas. (2006). Fundamentals of Critical Argumentation. New York: Cambridge University Press.

Walton, Douglas, Chris Reed \& Fabrizio Macagno (2008). Argumentation Schemes. New York: Cambridge University Press. 